\title{
SAXS and X-ray crystallographic studies of the assembly of the CARD promoter of the apoptosome
}

\author{
Su-Chang Lina, Tsung-Wei Sub and Yu-Chih Lo ${ }^{c}$
}

aGenomics Research Center, Academia Sinica, Taipei 11529, Taiwan, tomlin@gate.sinica.edu.tw ${ }^{\mathrm{b} G e n o m i c s ~ R e s e a r c h ~ C e n t e r, ~ A c a d e m i a ~ S i n i c a, ~ T a i p e i ~ 11529, ~ T a i w a n, ~ d a r r e n 0615 @ g m a i l . c o m ~}$ 'Department of Biotechnology and Bioindustry Sciences, College of Bioscience and Biotechnology, National Cheng Kung University, Tainan 70101, Taiwan, gracelo@mail.ncku.edu.tw

In the apoptotic signaling pathway, the human apoptosome is responsible for recruiting and subsequently activating procaspase- 9 . The recruitment of procaspase- 9 is mainly via the homotypic 1:1 CARD-CARD interaction between the Apaf-1 of the apoptosome and procaspase-9. How the recruitment could lead to the activation of procaspase-9 remains unclear.

Since the apoptosome contains seven Apaf-1 molecules, a higher order assembly between the Apaf- 1 and procaspase- 9 CARD, called the CARD-CARD disk, was expected. The first question that need to be answered is how the Apaf-1 and procaspase-9 CARDs assemble the CARD-CARD disk, especially on the heptameric apoptosome platform.

Here we solved the X-ray structure of the CARD-CARD assembly between Apaf-1 and procaspase-9 CARD and utilized SAXS data to help us elucidate the composition and assembling mechanism of the CARD-CARD disk (Figure 1).

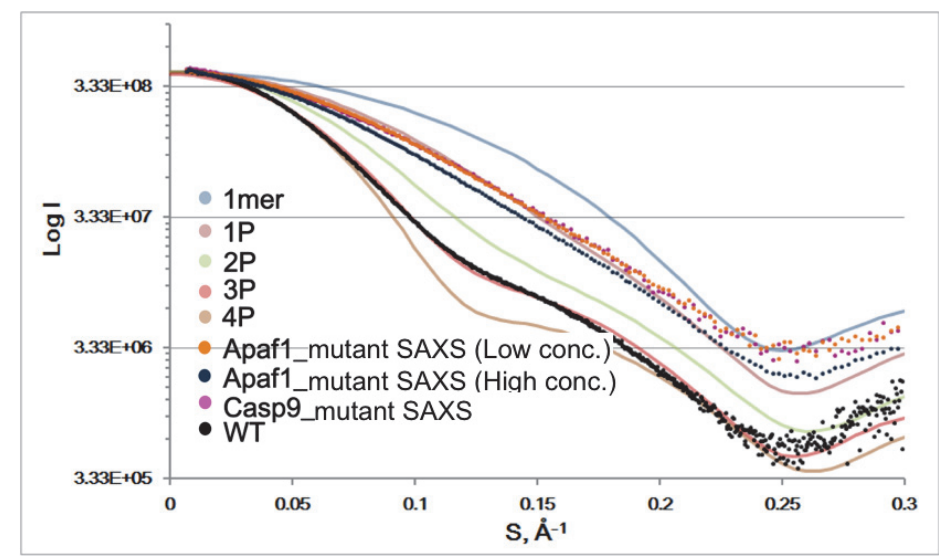

Figure 1. Comparison of the SAXS data with the curves from different models.

References

[1] Su, TW. et al. (2017) Structure. 25(3):407-420.

[2] Wang, L. et al. (2017) Structure. 25(4):575-577.

[3] Kumar, S. \& Dorstyn, L. (2017) F1000Prime. 10.3410/f.727231443.793531601 